DOI: http://dx.doi.org/10.18524/1810-4215.2017.30.114685

\title{
DOUBLE STATION OBSERVATION OF METEORS WITH LOW BASELINE IN MYKOLAIV
}

\author{
M.O.Kulichenko, O.V.Shulga \\ Research Institute «Mykolaiv Astronomical Observatory», \\ Mykolaiv, Ukraine,niiko4kulichenko@gmail.com,shulga-av@ukr.net
}

ABSTRACT. Results of single and double station observations of meteors using TV CCD techniques which were conducted in 2013-2016 in Mykolaiv astronomical observatory (RI MAO) are shown in the article.

Keywords: meteor, combined observational method, meteoroid heliocentric orbit.

\section{Introduction}

Meteor observational campaign using TV CCD unintensified techniques was started in 2013 in Mykolaiv astronomical observatory (RI «MAO») (Kulichenko et al., 2014 ; 2015). The main accent of the research is made on precise astrometry and meteoroid orbits calculation.

\section{Observation facilities}

System of meteor telescopes includes 6 optical telescopes (4 lenses: $f=85 \mathrm{~mm}, \mathrm{f} / 1.8 ; 2$ lenses: $\mathrm{f}=100 \mathrm{~mm}$, $\mathrm{f} / 2.0$ ) equipped with a TV CCD cameras WAT-902H2 $(768 \times 576,8.6 \times 8.3 \mu)$. The field of view of 4 telescopes is $3.2^{\circ} \times 4.2^{\circ}$ and $2.7^{\circ} \times 3.6^{\circ}$ for 2 telescopes. The length of baseline is $11.8 \mathrm{~km}$. Cameras work in the interlace mode with rate 50 half-frames per second for better time resolution. The observations were conducted in completely automatic mode using on-line meteor detection software developed at RI «MAO». The method of meteor registration is based on combined observation method (Shulga et al., 2008; 2011). Limiting magnitude for stars is in (11$12)^{\mathrm{m}}$. Mean accuracy of positional measurement for reference stars is (6-8)". The method of double station synchronization is based on using PPS-impulse from GPS receiver Resolution- $T$ as reference impulse. Accuracy of time synchronization is $10^{-4} \mathrm{~s}$.

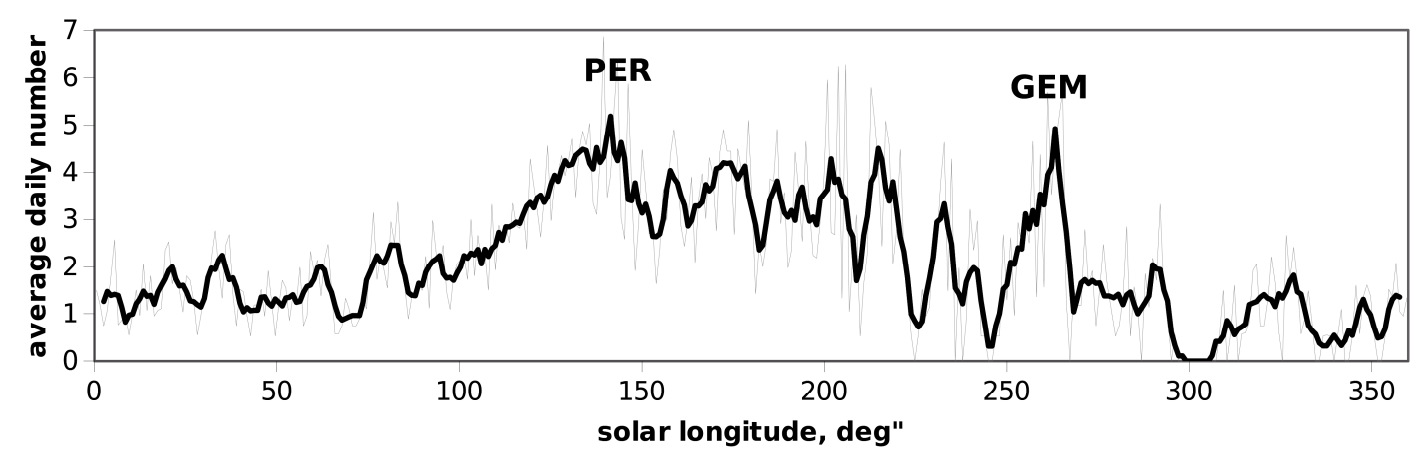

Figure 1: Meteor number variation per year. Averaged data 2013-2016

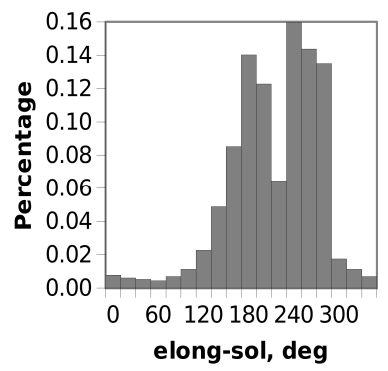

a)

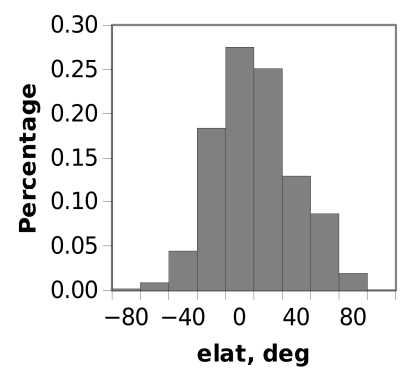

b)

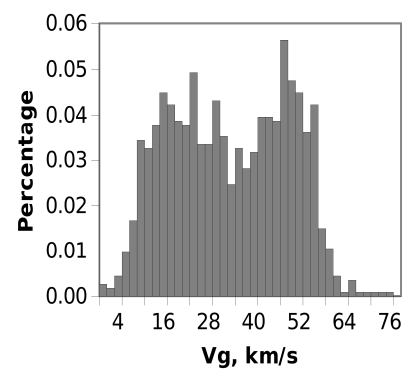

c)

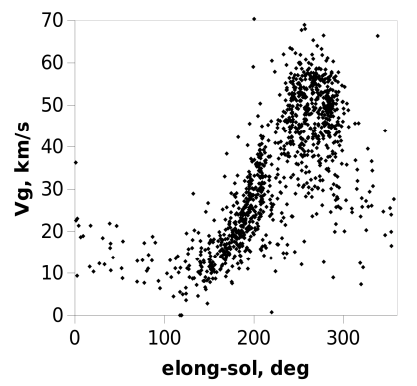

d)

Figure 2: Distributions of: a) ecliptic longitude of radiant position relative to solar longitude; b) ecliptic latitude of radiant position; c) geocentric velocity of meteors; d) diagram geocentric velocity - ecliptic longitude reduced to the solar longitude. 


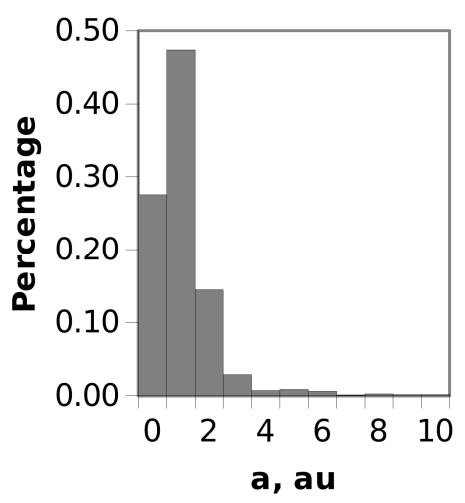

a)

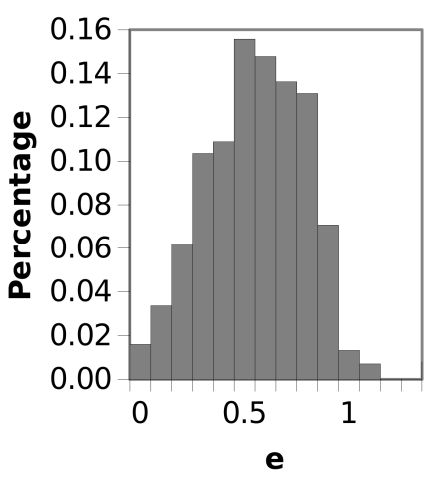

b)

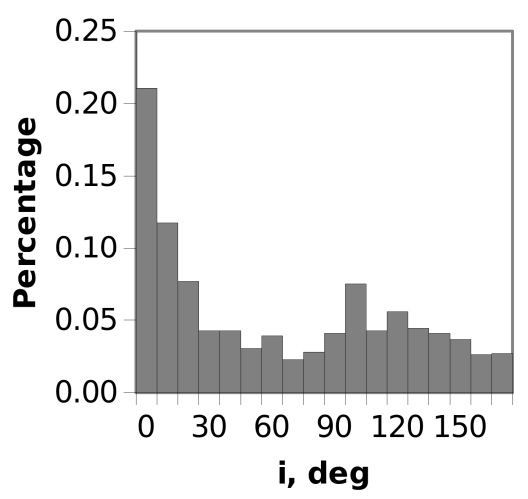

c)

Figure 3: Distribution of some meteoroid orbit elements: a) semi-major axis; b) eccentricity; c) inclination.

\section{Results}

\subsection{Single station observation}

In 2013-2016 9110 single station meteors were registered. Due to weather conditions, narrow fields and technical problems the meteor quantity distribution over solar longitude for single camera is not representative. However it is possible to obtain average smoothed annual variation using sum of all 6 cameras observations for 3 years (Fig. 1).

This single station statistics shows increase of meteors number in the second part of year and strict peaks of main meteor showers such as Perseid and Geminid. The mean duration of observed meteor trajectories is in 0.05-0.6 s. The distributions of meteors over meteor magnitude and arc length have their maxima at $2^{\mathrm{m}}$ and $(0.75-1)^{\circ}$ and include meteors with range from $-2^{\mathrm{m}}$ to $7^{\mathrm{m}}$. $85 \%$ of meteor trajectories are partial because of narrow field of view but due to high time resolution $>60 \%$ of detected trajectories have $>5$ frames.

\subsection{Double station observation}

In 2013-16 1148 double station meteors were observed. Several meteor showers both major (Perseid, Geminid etc) and minor (e.g. Southern delta Aquariids) were matched. More than $80 \%$ of meteors are sporadic.

Double station trajectories have atmospheric path length range in $1.2-15 \mathrm{~km}$ with maximum at $4-5 \mathrm{~km}$. Atmospheric heights range is in $60-120 \mathrm{~km}$. The average accuracy of visible radiant estimation is less $0.5^{\circ}$ with baseline $11.8 \mathrm{~km}$. The accuracy of geocentric velocity estimation is $0.5 \mathrm{~km} / \mathrm{s}$. Maximum of heliocentric radiants (ecliptical coordinates of radiant) distribution is near the ecliptic plane (Fig. 2, b). Heliocentric radiants distribution has two typical maximums near the apex point and Antihelion source point which are most power sources of sporadic meteors in optical range (Fig. 2, a). Geocentric velocity distribution has two peaks at 15 and $50 \mathrm{~km} / \mathrm{s}$ which is corresponding with heliocentric radiant distribution: first peak is average velocity of sporadic meteors from Antihelion source, second peak belongs to sporadic meteors from apex (Fig. 2, c, d). Orbital elements distributions show that most of meteoroid orbits belong to near Earth object with eccentricities $0.5-0.8$ and have low inclinations (Fig. 3, a-c). Local maximum in inclination distribution near $110^{\circ}$ corresponds with Perseid meteor shower.

\section{Conclusion}

Based on the results of meteor observation in 20132016 catalog containing heliocentric orbital elements of meteoroids and kinematic parameters for their atmospheric path has been obtained.

\section{References}

Kulichenko N., Shulga O., Kozyryev Y. et al.: 2014, in Gyssens M., Roggemans P., Zoladek, P., eds., PIM3, 50. (2014pim3.conf...50K)

Kulichenko N., Shulga O., Kozyryev Y. et al.: 2015, JIMO, 43, 81. (2015JIMO ...43...81K)

Shulga O., Kozyryev Y., and Sybiryakova Y.: 2008, in IAU Symp., 248, 128. (2008IAUS..248..128S)

Shulga O., Kozyryev Y., and Sybiryakova Y.: 2011, in Tanga P., Thuillot W., eds., GFUN, 97. (2011gfun.conf...97S) 